ORIGINAL ARTICLE

\title{
Dynamic Ultrasound in Morphological Assessment of Medial Compartment of
} Knee Joint Osteoarthritis

\author{
Dalia Eid Bishr*, Magdy Abbas El-Fawal, Khaled Mohamed El-Gerby , Ahmed Mohamed El- \\ Maghraby \\ Radio diagnosis Department, Faculty of Medicine, Zagazig University. Zagazig, Egypt
}

\author{
*Corresponding \\ author: Dalia Eid Bishr \\ Radio diagnosis \\ Department, Faculty of \\ Medicine, Zagazig \\ University. Zagazig, \\ Egypt
}

E-mail :

dalia.3eed@gmail.com

Submit Date 2019-01-19

Revise Date 2019-04-01

Accept Date 2019-04-08

\section{ABSTRACT}

Background: Osteoarthritis is a major joint disease between the elderly with the knee is the most common joint involved. Ultrasound is used for non-invasive assessment of knee joint status with evaluation of the meniscal position, joint space narrowing and presence of osteophytic lipping under load-bearing position for early detection of osteoarthritis. The aim of the study is to assess the role of high-resolution ultrasonography with dynamic assessment in evaluation of the medial compartment knee joint osteoarthritis.To investigate the correlation between the O.A grade determined by radiography and that by ultrasound evaluation and to investigate the diagnostic accuracy of O.A determined using US. Methods: This study was carried out at Radio diagnosis Department, Zagazig University Hospitals. The present study was carried on 35 patients of knee osteoarthritis. Diagnostic work up was done including plain radiography \& MSUS. Results: Total of 38 of the 40 knees were correctly diagnosed by ultrasonography with sensitivity of $95 \%$ and specificity of $80 \%$. The predictive value positive was $86.36 \%$ and the predictive value negative was $92.3 \%$ with $88.5 \%$ accuracy. Conclusions: Our results indicate that knee ultrasonography provides valuable information for the assessment of knee joint osteoarthritis. However, MSUS has an advantage of dynamic evaluation with qualitative and semi-quantitative assessment for early detection of knee O.A with good diagnostic accuracy.

Keywords: Knee joint; Osteoarthritis; Dynamic Ultrasound

\section{INTRODUCTION}

$\mathrm{O}$ steoarthritis (OA) is the most prevalent chronic joint disease, impacting many joints including knees, hands, hips and spine. It's considered a main source of chronic pain with impaired mobility in [1] .

Knee osteoarthritis (KOA) is responsible for $80 \%$ or more of the disease's total burden. It's prevalence is increasing in aging populations worldwide, as it's reported that it has been doubled in prevalence since the mid- $20^{\text {th }}$ century [2].

Knee OA is a multi-factorial disease including the effect of aging, sex, body mass index (BMI), genetic predisposition and mechanical influences such as meniscal lesions, joint malalignment and previous injury or surgery. It is September 2019 Volume 25 Issue 5 mainly manifested by knee pain, stiffness and joint instability that direct the patient to seek medical attention [1].

$\mathrm{KOA}$ is no longer considered as a "wear and tear" disease, but a whole-organ disorder that involves multiple structures of the joint. The disease process includes progressive articular cartilage degeneration, osteophytes formation, areas of abnormal sub-chondral bony sclerosis, structural changes in bone trabeculae and bone marrow, inflammatory infiltration of the synovium and loosening as well as weakness of muscles and tendons [3, 4].

Traditionally, knee OA is primarily diagnosed by conventional radiography using KallgrenLawrence (KL) grading by structural changes such as osteophyte presence and joint space www.zumj.journals.ekb.eg 
narrowing (JSN) which mainly appear at relatively late stages [3].

Although in the recent decades magnetic resonance imaging (MRI) has become the gold standard imaging modality for different knee pathologies, it's not usually used as an initial imaging technique for knee OA due to practical and cost reasons. Recently, high resolution ultrasound possesses a high potential in assessment of knee OA among other imaging modalities as it allows qualitative and quantitative assessment of the articular cartilage and plays an important role in screening and follow-up. (3).

Having the advantages of being safe, available with no ionizing radiations, in addition to being real-time allowing direct visualization of structural changes in cartilage and menisci make high resolution ultrasound superior among other imaging modalities (2).

Unlike radiography, ultrasound can detect early OA morphological degeneration while conventional radiography reveals minor doubtful changes (4) as it is much more sensitive to detect osteophytes 3 ), more reliable to assess meniscal extrusion (5) and allows direct assessment of changes in soft tissues. In addition it is useful to evaluate the rapid progression of OA changes in patients with OA seen on radiographs (6).

\section{METHODS}

In this cross-sectional study, consecutive patients were clinically diagnosed in Orthopedic and Rheumatology outpatient clinic with primary knee OA. Written informed consent was obtained from all participants and the study was approved by the research ethical committee of Faculty of Medicine, Zagazig University. The work has been carried out in accordance with The Code of Ethics of the World Medical Association (Declaration of Helsinki) for studies involving humans

- Patient selection: 35 patients (4 males and 31 females, 70 knees) with knee OA referred to radiology department of Zagazig University Hospitals for ultrasonographic examination were recruited during the period from February 2018 to August 2018. Their ages ranged between 45-69 years.

- Inclusion criteria of the patients:

- Patients complain of knee joint pain and under clinical suspicion of knee OA.

- Exclusion Criteria: Any subject with history of:

- Trauma

- Infection \& inflammatory condition e.g. RA

- Neuropathic arthropathy, Gout or pseudo gout.

- Patients with history of surgical treatment

- Patients unable to stand during ultrasonographic examination \& unable to perform knee flexion or could not extend their knees completely.

Method: All patients meeting the previously mentioned inclusion and exclusion criteria were subjected to the following

$>$ Full clinical history: Personal history (name, age, sex, occupation). Present history: The main clinical presentation of these patients. Past history of trauma or operation.

$>$ Radiological assessment:

- Conventional radiography.

- Ultrasound examination.

\section{Conventional radiography:}

Conventional plain $\mathrm{x}$-ray was taken of the target knee in the antro-posterior (AP) view with the patient in a standing position. The radiograms were obtained using Fuji system.

Image interpretation: The images were reviewed for the following items: Narrowing of the medial compartment (2) osteophytes lipping (3) sclerosis (4) subchondral cysts (5) Deformities of bone. And the image was given a score according to KL score (KELLGREN \& LAWRENCE, 1957).

Ultrasound assessment of the medial compartment structures of the knee:

All patients had standardized ultrasonography of the knee joint with excess gel was used instead of the gel pad. Ultrasound 
examinations were performed using GE Logic probe $(7-11 \mathrm{MHz})$.

Dynamic US was used to examine the medial knee joint to diagnose the medial radial displacement (MRD) of the medial meniscus and the peripheral joint space (PJS). US examinations were performed with both knees in complete extension in the supine position then the patient weights on the diseased knee to examine the affected knee joint in the standing position. The MRD and PJS were measured on longitudinal US images at the site at which the medial collateral ligament (MCL) was most clearly visualized. Normal MCLs are observed to be two hyperechoic bands separated by a thin hypoechoic zone crossing over the medial femoral condyle, the margin of the medial meniscus and the medial tibial plateau. The deeper layer closely adheres to the peripheral edge of the meniscal body, but in OA, where the meniscus is frequently extruded and macerated and the MCL is displaced radially, there is insufficient contrast between the two structures in US. The MRD was measured as the distance from the outer most edge of the medial meniscus to a line connecting the femoral and tibial cortices. The PJS was measured as the distance from the peripheral femoral to the peripheral tibial cortices. When marginal osteophytes were present in the femur and tibia, the MRD and the PJS were measured on the clearly visualized normal cortical bone continuous with the femoral and tibial marginal osteophytes. The distances were measured using electronic calipers (to an accuracy of $0.1 \mathrm{~mm}$ ) Fig.(1)

We defined the supine position MRD and PJS as the non-weight- bearing medial radial displacement (NWMRD) and nonweight-bearing peripheral joint space (NWPJS), respectively, and the standing position MRD and PJS as the weight-bearing medial radial displacement (WMRD) and weight-bearing peripheral joint space (WPJS), respectively. The average duration time of the examination was from 15-20 minutes.

\section{Statistical Analysis:}

September 2019 Volume 25 Issue 5
All data were collected, tabulated and statistically analyzed using SPSS 20.0 for windows (SPSS Inc., Chicago, IL, USA). Quantitative data were expressed as the mean \pm SD \& (Minimum-maximum), and qualitative data were expressed as absolute frequencies (number) \& relative frequencies (percentage). Student's t-test was used to compare between two groups of normally distributed variables. Anova $(F)$ test was used to compare between more than two independent groups of normally distributed variables. LSD (least significant difference) of PAIRWISE between each group. All tests were two sided. p-value $<0.05$ was considered statistically significant $(\mathrm{S})$, and $\mathrm{p}$ value $\geq 0.05$ was considered statistically insignificant (NS).

\section{RESULTS}

Our study includes 70 symptomatic knees from 35 patients. Age of the patients ranged from 45 to 69 years with mean age $57 \pm 7.7$.

Using knee radiographs, the patients are divided into five groups according to K-L grading with $5.7 \%$ of knees have grade 4 lesion as severe degree of knee $\mathrm{OA}$ as shown in table(1).

Ultrasound examination of the studied group shows that the mean of NW_MRD is $6.9 \mathrm{~mm}$ with its minimum value is 4.1 , maximum value is $13.3 \mathrm{~mm}$. In the standing position the mean of W_MRD is $8.1 \mathrm{~mm}$ with its minimum value is 4.4 , maximum value is $14.8 \mathrm{~mm}$. Also, the mean of the NW-PJS was $3.8 \mathrm{~mm}$ with its minimum value is $1.6 \mathrm{~mm}$, maximum value is $9.2 \mathrm{~mm}$ while in the standing position W-PJS mean was $2.9 \mathrm{~mm}$ with its minimum value is $1.5 \mathrm{~mm}$, maximum value is $6.2 \mathrm{~mm}$. Joint effusion was found in $67 \%$ of the subjects while Backer's cyst in $11.4 \%$ only Table (2).

No significant difference was noted in the MRD without weight-bearing between the KL grade 0 and $\mathrm{KL}$ grade 1 knees, but significant difference appears in the weight-bearing position. There's significant difference between KL grade 0 and KL grade 2 or more severe grade knees regarding NW-MRD and W-MRD values. There's significant difference between 
grade 1,2 and 3 knees with higher grades regarding the MRD with and without weightbearing positions $(\mathrm{P}<0.0001)$ Table $(3)$.

With regard to PJS, there's significant difference between grade 0 and more higher grades in both supine and standing positions. No significant difference was noted between grade 1 and 2 in the PJS with and without weight-bearing positions yet, significant differences appear between KL grade 1 and grade 3. NWPJS values were significantly indifferent between KL grade 1 and grade 4 and between KL grade 2 and grade 3 or 4 knees with significant difference shown in the weightbearing positions. No significant difference noticed between KL grade 3 and 4 regarding PJS in the weight and non-weight bearing positions Table (3).

Additionally, we divide the subjects into two groups, KL grade 0 and 1 as non-osteoarthritic group (non-OA group) while KL grade 2-4 as osteoarthritic group (OA group). $57.14 \%$ of the examined knees affected by osteoarthritis Table (4).
The mean value of the NWMRD in the non-OA group was $5.13 \mathrm{~mm}$, while that in the OA group was $8.19 \mathrm{~mm}$. The mean value of the WMRD in the non-OA group was $5.9 \mathrm{~mm}$, while that in the OA group was $9.68 \mathrm{~mm}$. The NWMRD and WMRD values were significantly lower in the non-OA group than in the OA group $(p<0.0001)$.The mean values of NWPJS and WPJS in the non-OA group were $4.4 \mathrm{~mm}$ and $3.6 \mathrm{~mm}$ respectively, while those in the OA group were $3.4 \mathrm{~mm}$ and $2.5 \mathrm{~mm}$, respectively. The NWPJS and WPJS values were both significantly higher in the non-OA group compared to the OA group ( $\mathrm{p}<0.001)$ Table (5). When we defined OA by ultrasound as WMRD $>5 \mathrm{~mm}$ and W-PJS $<5 \mathrm{~mm}$, total of 38 of the 40 knees were correctly diagnosed by ultrasonography with sensitivity of $95 \%$ and specificity of $80 \%$. The predictive value positive was $86.36 \%$ and the predictive value negative was $92.3 \%$ with $88.5 \%$ accuracy Table (6).

Table 1. Frequency distribution of X-ray grade of studied knees.

\begin{tabular}{|l|l|l|}
\hline X-ray grade & No & $\%$ \\
\hline Grade 0 & 12 & 17.2 \\
\hline Grade 1 & 18 & 25.7 \\
\hline Grade 2 & 18 & 25.7 \\
\hline Grade 3 & 18 & 25.7 \\
\hline Grade 4 & 4 & 5.7 \\
\hline
\end{tabular}


Table 2. Ultrasound finding of studied group.

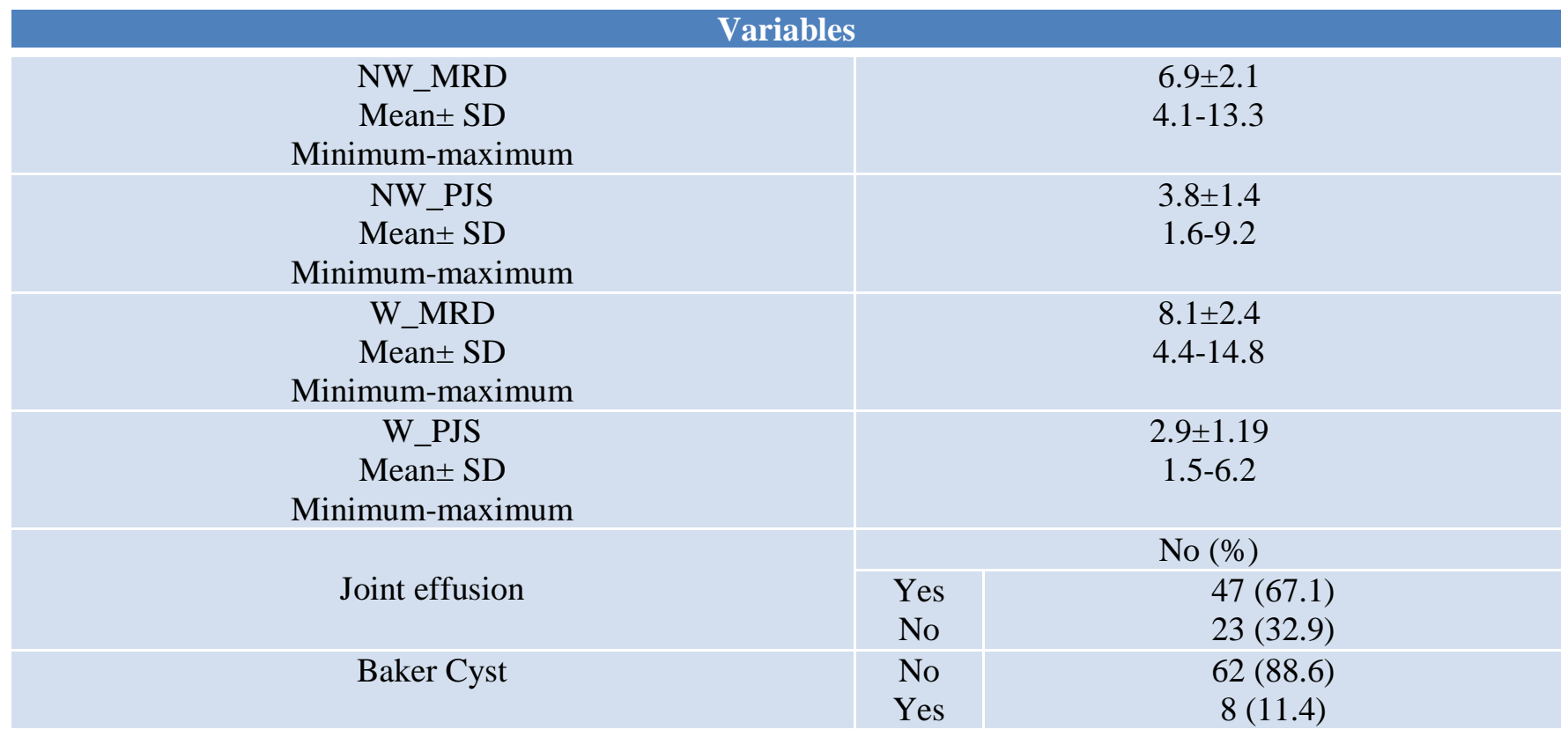

Table 3. Comparison between each x-ray grade and others regards NW_MRD, NW_PJS, W_MRD and W_PJS.

\begin{tabular}{|c|c|c|c|c|c|}
\hline \multicolumn{2}{|c|}{ X-ray grade } & NW-MRD & NW-PJS & W-MRD & W-PJS \\
\hline \multirow[t]{4}{*}{ Grade 0} & Grade1 & 0.062 & 0.02 & .041 & 0.001 \\
\hline & Grade2 & 0.0001 & 0.006 & 0.0001 & 0.0001 \\
\hline & Grade3 & 0.0001 & 0.0001 & 0.0001 & 0.0001 \\
\hline & Grade4 & 0.0001 & 0.003 & 0.0001 & 0.0001 \\
\hline \multirow[t]{3}{*}{ Grade1 } & Grade2 & 0.0001 & 0.7 & 0.0001 & 0.6 \\
\hline & Grade3 & 0.0001 & 0.049 & 0.0001 & 0.002 \\
\hline & Grade4 & 0.0001 & 0.13 & 0.0001 & 0.007 \\
\hline \multirow[t]{2}{*}{ Grade2 } & Grade3 & 0.0001 & 0.11 & 0.0001 & 0.01 \\
\hline & Grade4 & 0.0001 & 0.2 & 0.0001 & 0.02 \\
\hline Grade3 & Grade4 & 0.0001 & 0.7 & 0.0001 & 0.4 \\
\hline
\end{tabular}

Table 4. Frequency distribution of osteoarthritis of studied knees.

\begin{tabular}{|l|l|l|}
\hline Variables & No. & $\%$ \\
\hline Osteoarthritis & 40 & 57.14 \\
\hline No osteoarthritis & 30 & 42.86 \\
\hline
\end{tabular}


Table 5. Comparison of NW_MRD, NW_PJS, W_MRD and W_PJS regard osteoarthritis of studied knees.

\begin{tabular}{|c|c|c|c|c|}
\hline & $\begin{array}{c}\text { osteoarthritis } \\
\text { present } \\
\mathrm{N}=40\end{array}$ & $\begin{array}{c}\text { Osteoarthritis } \\
\text { absent } \\
\mathbf{N}=\mathbf{3 0}\end{array}$ & $\mathbf{T}$ & $\mathbf{p}$ \\
\hline & $\begin{array}{l}\text { Mean } \pm \text { SD } \\
\quad(\mathrm{mm})\end{array}$ & $\begin{array}{l}\text { Mean } \pm \text { SD } \\
\quad(\mathrm{mm})\end{array}$ & & \\
\hline NW_MRD & $8.19 \pm 1.87$ & $5.13 \pm 0.8$ & 9.3 & $0.0001(\mathrm{~S})$ \\
\hline NW_PJS & $3.4 \pm 1$ & $4.4 \pm 1.6$ & 3.2 & $0.002(\mathrm{~S})$ \\
\hline W_MRD & $9.68 \pm 1.8$ & $5.9 \pm 1$ & 10 & $0.0001(\mathrm{~S})$ \\
\hline W_PJS & $2.5 \pm 0.85$ & $3.6 \pm 1.3$ & 4.2 & $0.0001(\mathrm{~S})$ \\
\hline
\end{tabular}

Table 6. Contingency table showing the results of the ultrasonography and radiographic evaluations of OA in the knee.

\begin{tabular}{|l|l|l|l|} 
& $\begin{array}{l}\text { Positive } \\
\text { Radiography } \\
\text { (No.) }\end{array}$ & $\begin{array}{l}\text { Negative } \\
\text { Radiography } \\
(\text { No.) }\end{array}$ & $\begin{array}{l}\text { Total } \\
\text { (No.) }\end{array}$ \\
\hline Positive Ultrasound (No.) & 38 & 6 & 44 \\
\hline Negative Ultrasound (No.) & 2 & 24 & 26 \\
\hline Total (No.) & 40 & 30 & 70 \\
\hline
\end{tabular}

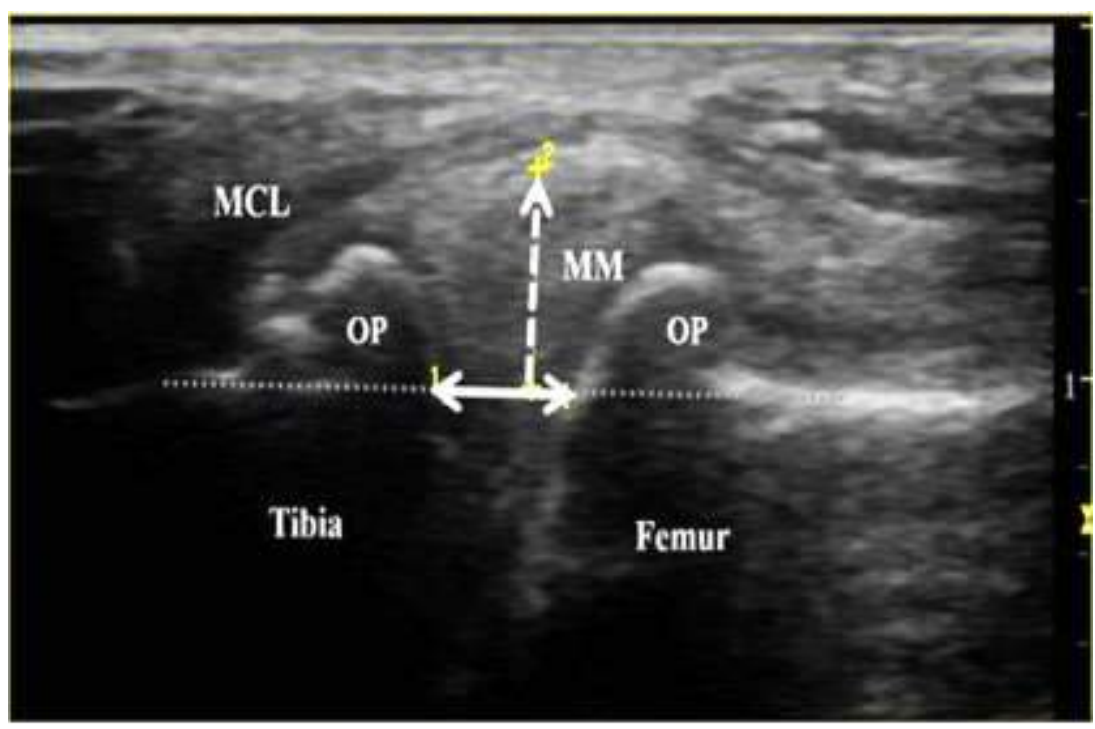

Figure 1A: The MRD and PJS were measured on longitudinal US images at the site at which the medial collateral ligament (MCL) was most clearly visualized. $\mathrm{OP}=$ osteophyte, $M M=$ medial meniscus, $M C L=$ medial collateral ligament, double-sided arrow peripheral joint space. ${ }^{9}$ 


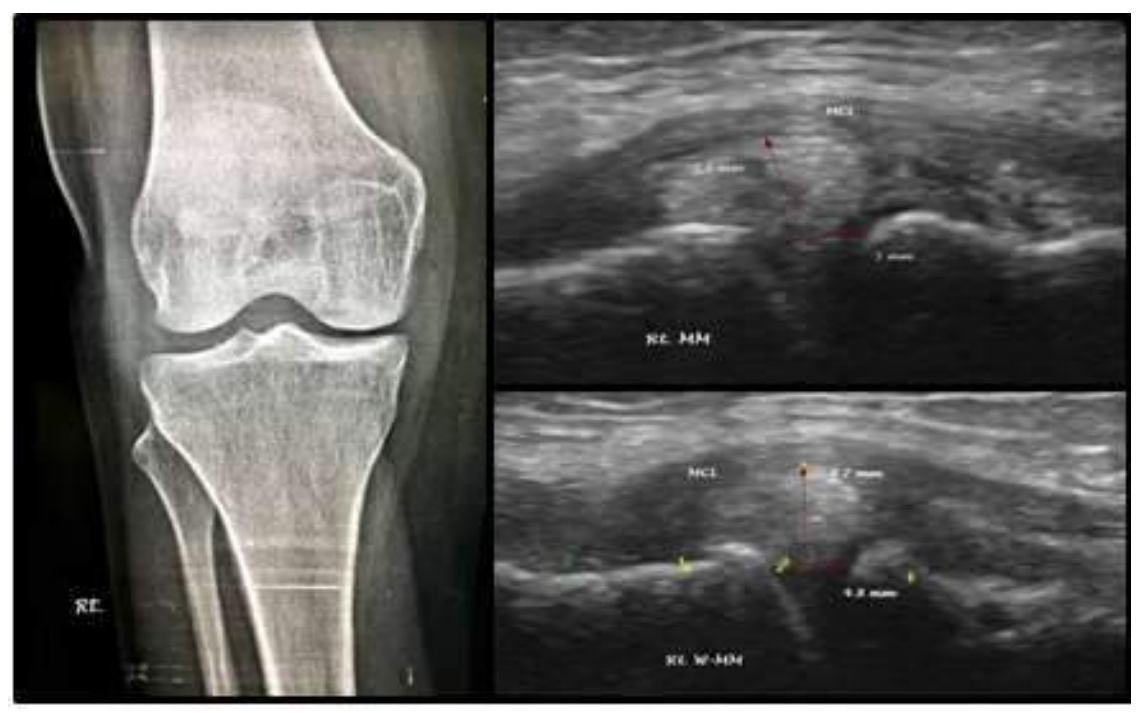

Figure 1B. Rt. Knee radiograph AP view (Rt. Sided image) KL grade1 (non-osteoarthritis), US of medial compartment (supine position)(top left) $\mathrm{NW}$-MRD=5.8 mm, NW-PJS=5 mm, US of medial compartment (weight-bearing)(bottom left) WMRD $=6.7 \mathrm{~mm}, \mathrm{NW}-\mathrm{PJS}=4.8 \mathrm{~mm}$ (Osteoarthritis).

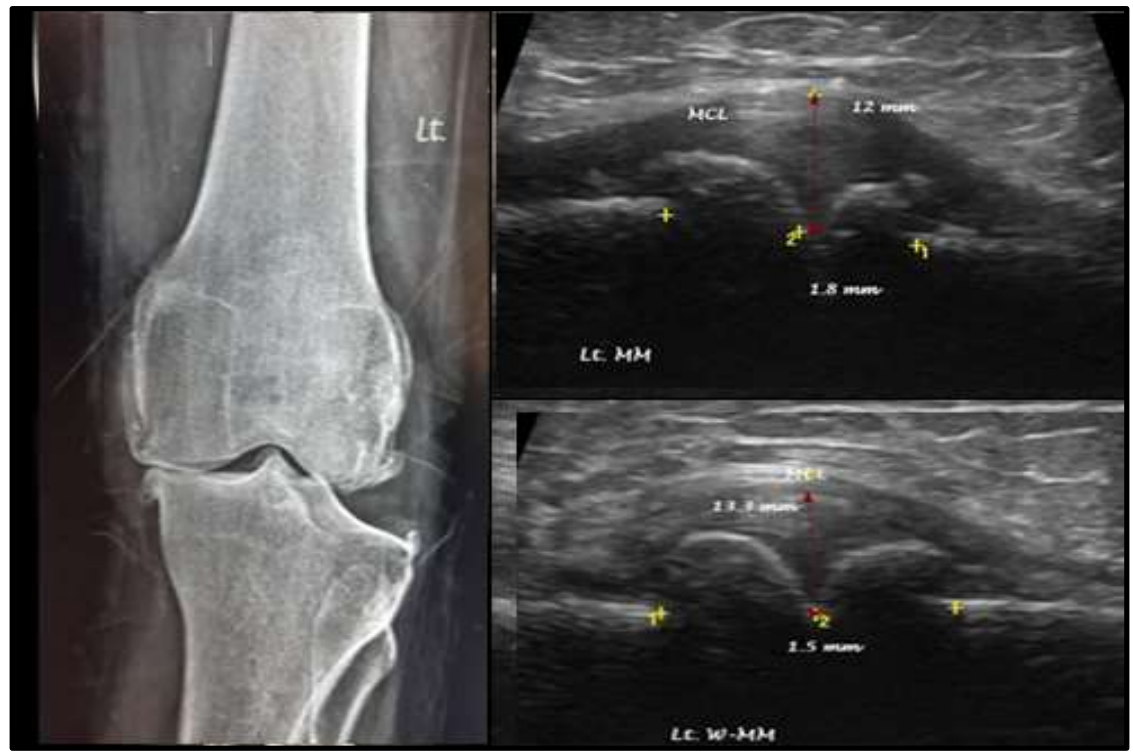

Figure 1C. Lt. Knee radiograph AP view (Rt. Sided image) KL grade4 (osteoarthritis), US of medial compartment (supine position)(top left) $\mathrm{NW}-\mathrm{MRD}=12 \mathrm{~mm}$, NW-PJS=1.8 mm with osteophytic lipping, US of medial compartment (weightbearing)(bottom left) W-MRD=13.3mm, W-PJS=1.5 mm, osteophytes become more prominent(Osteoarthritis).

\section{DISCUSSION}

Knee OA is a common disease which is more frequent in the medial compartment than in the lateral compartment. During normal gait, there is initial valgus moment followed by external varus throughout the stance phase. This external varus provides subsequent increased loads in the medial compartment which is thought to be accountable for the higher incidence of osteoarthritis in the medial compartment [9]. The KL grade is simply a plain radiological diagnosis, and is commonly accepted by radiologists and clinicians as a standard grading system for knee joint osteoarthritis. However, the KL scale cannot characterize cartilage damage, including that of the meniscus, so the medial radial displacement 
(MRD) cannot be detected by radiography, while it can be examined by US. This suggests that US can provide additional information about the severity of OA that cannot be provided by radiography [10].

Ultrasonography (US) has become widely used with great interest in knee OA. Being fast, non-invasive with lack of ionizing radiations and no contrast, no sedation or contraindications from claustrophobia or mental implants are all clear advantages that rise ultrasound validity [11]. The additional value of ultrasound is its ability to directly visualize internal structural changes. US can evaluate structural changes in cartilage, tendons, synovitis, bursitis as well as bony changes related to OA like subchondral bone and osteophytes. Early detection of meniscal extrusion is relatively important as knee OA can be suggested by articular cartilage loss which occurs secondary to meniscal extrusion [12].

Ultrasound offers a relatively simple way to assess meniscal extrusion with the patient both supine (at rest) and standing (weight bearing), therefore it could be applied to the assessment of static and dynamic displacement of menisci with precise localization of tissue damage [5].

This study was performed to diagnose and evaluate medial type of the knee osteoarthritis (OA) using high resolution dynamic ultrasound (US).

Our study included 35 patients with 70 knees whom were referred to radiodiagnosis department for ultrasound examination in the study period. They were diagnosed to have degenerative arthritis of the knee joint with age range from 45-69 years old and about $88.6 \%$ were females.

In this study, the majority of the patients $(88.6 \%)$ were females and the minority of the patients $(11.4 \%)$ was males. This was in line with Cubukcu et al. [13] who reported that the number of female patients with knee OA is striking. This condition could be partly explained in aging women with the differentiation of the hormonal status and the imbalance in the formation and destruction of bone. Menopause has been associated with an increased production of interleukin-1 which is the part of the cytokine response in OA. In postmenopausal women as the level of estrogen decreases, interleukin-1 levels can increase which leads to OA. Also, other issues like anatomical differences, previous trauma and genetic effect should be considered.

Since medial compartment $\mathrm{OA}$ is more prevalent in people with knee OA and the MCL can be easily identified by MSK ultrasonography with good reproducibility, our study was designed to measure meniscal displacement of the medial meniscus at the level of the MCL. We believe that measuring displacement at the level of the MCL alone may overlook meniscal displacement in other directions, and similar displacements may occur in the lateral meniscus but were not estimated in this study.

Although MRI is an excellent imaging modality for evaluating meniscal abnormalities and its position with semiquantitative and quantitative assessments, images are obtained routinely in a supine, non-weight bearing position. In addition, MRI is relatively costly and requires considerable time to finish the examination [5]. Zhang et al [13] defined meniscal extrusion as a pathological condition in which the outer surface of the meniscus exceeds the surface of the tibia by more than $3 \mathrm{~mm}$. Analysis of (15) ROC curve revealed that the cut-off value of medial meniscal extrusion for knee pain was $4.3 \mathrm{~mm}$ with sensitivity $84.5 \%$ and specificity $85 \%$.

In agreement to his study, all our cases were symptomatic complaining of knee pain mainly and to lesser extent other symptoms with the least MRD found was $4.4 \mathrm{~mm}$ in the weight bearing position. 
In the present study, pain was the most common presentation and there was high significant relation between the K-L grading scale and the disease activity parameters including pain, stiffness, and reduced function. This was in agreement with previous studies of Cubukcu et al. [13] and Finan et al. [15] as they stated that pain is the main complaint among patients with knee OA and a leading cause of physical disability.

Although, individuals with radiographic evidence of OA may be asymptomatic at any time, radiographic features of OA were significantly associated with knee pain. It is possible that pain bears a stronger relationship to radiographic features in patient with severe disease. On the other hand, CR which is the most commonly used imaging modality may not identify bony changes related to pain in early knee OA unlike US. Radiographs demonstrate structural changes rather than disease severity as it permits only limited assessment of the knee compartments provides only an approximation of articular cartilage change with measurement of joint space narrowing, and poorly characterizes other soft tissues [3]. Medial meniscus extrusion detected by ultrasound, similar to that measured by MRI has been shown to be linked to the development and progression of knee osteoarthritis [15].

In agreement with, Kawaguchi et al [16] who measured the medial radial displacement (MRD) of the medial meniscus with US with increased displacement in the weight bearing, we found a close association between extra-articular extrusion of the medial meniscus and progression of OA.

Similarly Chiba et al [6] in his 3-year prospective study showed that extruded medial meniscus seen on ultrasonography is associated with development of knee osteoarthritis with more JSN and osteophyte formation seen on radiography at 3-year follow up even after adjusting age, sex, symptoms and knee radiographic findings at the baseline.

Likewise, in our study, meniscal displacement was found to be significant in symptomatic OA cases in both weight bearing and non-weight bearing positions.

In our study, US parameters between osteoarthritic and non-osteoarthritic groups were significantly different. Our results showed that the non-weight bearing medial radial displacement (NWMRD) and the weight bearing medial radial displacement (WMRD) increased in subjects with OA than non-OA $(\mathrm{P}<0.0001)$. The non-weight bearing peripheral joint space (NWPJS) and weight bearing peripheral joint space (WPJS) decreased in OA than in non-OA $(\mathrm{P}<0.002)$ with more significant decrease in the weight bearing position $(\mathrm{P}<0.0001)$.

In agreement to our results Yanagisawa and his colleagues (A) in 2014 reported The NWMRD and WMRD values were significantly lower in the non-OA group than in the OA group $(\mathrm{P}<0.001)$. The NWPJS and WPJS values were both significantly higher in the non-OA group compared to the OA group $(\mathrm{P}<0.001)$.

Our findings confirm that the degree of displacement is significantly higher in the weight-bearing standing position than in the non-weight-bearing supine position (dynamic displacement) $(\mathrm{P}<0.0001)$.

Similarly to Yanagisawa and his colleagues [4], we found that the degree of joint space narrowing is significantly higher in the weight-bearing standing position than in the non-weight-bearing supine position $(\mathrm{P}<$ 0.007).

As knee OA diagnosed by radiography in the standing position, we use ultrasound values measured in the weight-bearing position for comparison.

We use ultrasound parameters proposed by Yanagisawa et al [4] who classified patients who met the following criteria as having OA 
on the US assessment: > $5 \mathrm{~mm}$ of MRD during weight-bearing, $<5 \mathrm{~mm}$ of PJS during weight-bearing, and $>2 \mathrm{~mm}$ of osteophytes with $90.8 \%$ sensitivity $95.5 \%$ specificity.

Our results showed that ultrasound correctly diagnosed knee osteoarthritis in 38 knees with sensitivity of $95 \%$, specificity of $80 \%$, The predictive value positive was $86.36 \%$ and the predictive value negative was 92.3 $\%$ with accuracy reaching $88.5 \%$.

Six of the knees showed positive ultrasound parameters yet no signs of radiographic knee osteoarthritis and diagnosed as KL grade 1 . These knees suggest early appearance of OA as dysfunction of the meniscus with no appreciable structural bony changes or remarkable osteophytes. On the other hand, two knees showed normal ultrasound parameters although the mild osteoarthritic appearance on knee radiographs. This suggested normal meniscal appearance and function with normal joint space despite the early osteophytic lipping.

Therefore, ultrasound provides a good screening tool for knee OA together with knee radiographs as the results were accurate and reliable especially for early detection of knee OA.

There are some practical limitations, as we examined only the medial compartment of the knee being the most prevalent compartment for knee OA, we did not evaluate lateral and patellofemoral compartments in this study. Also, ultrasound evaluation at the MCL level may miss the displacement in other direction. Another limitation of the study design is the relatively small study group.

\section{CONCLOSION}

We conclude that assessment of medial meniscal extrusion with ultrasound is reliable and can be easily used for both qualitative and semi-quantitative assessment with good diagnostic accuracy.

\section{Declaration of interest}

The authors report no conflicts of interest. The authors alone are responsible for the content and writing of the paper.

\section{Funding information}

None declared

\section{REFERENCES}

1. Xia B, Di Chen D, Zhang J, Hu S, Jin H, Tong P. Osteoarthritis pathogenesis: a review of molecular mechanisms. Calcif Tissue Int. 2014;95(6):495-505. doi:10.1007/s00223014-9917-9

2. Wallace IJ, Worthington S, Felson DT, et al. Knee osteoarthritis has doubled in prevalence since the mid-20th century. Proc Natl Acad Sci $U \quad S \quad$ A. 2017;114(35):9332-9336. doi:10.1073/pnas.1703856114

3. Podlipská J, Guermazi A, Lehenkari P, et al. Comparison of Diagnostic Performance of Semi-Quantitative Knee Ultrasound and Knee Radiography with MRI: Oulu Knee Osteoarthritis Study. Sci Rep. 2016;6:22365. doi:10.1038/srep22365

4. Carotti M, Salaffi F, Di Carlo M, Giovagnoni A. Relationship between magnetic resonance imaging findings, radiological grading, psychological distress and pain in patients with symptomatic knee osteoarthritis. Radiol Med. 2017;(0123456789). doi:10.1007/s11547-017-0799-6

5. Nogueira-Barbosa, M. H., Gregio-Junior, E., Lorenzato, M. M., Guermazi, A., Roemer, F. W., Chagas-Neto, F. A et al., (2015): Ultrasound Assessment of Medial Meniscal Extrusion: A Validation Study Using MRI as Reference Standard. American Journal of Roentgenology, 204(3), 584-588.

6. Chiba D, Maeda S, Sasaki E, et al. Meniscal extrusion seen on ultrasonography affects the development of radiographic knee osteoarthritis: a 3-year prospective cohort study. Clin Rheumatol. 2017. doi:10.1007/s10067-017-3803-6

7. Petscavage-Thomas J. Clinical applications of dynamic functional musculoskeletal ultrasound. Reports Med Imaging. 2014;7:27. doi:10.2147/RMI.S40194

8. Kellgren JH, Lawreence JS. Radiological assessment of osteo-arthrosis. Ann Rheum Dis. $\quad 1957 ; 16(4): 494-502$. 
doi:10.1136/ard.16.4.494

9. Ko C, Chan K, Peng H. Sonographic Imaging of Meniscal Subluxation in Patients with Radiographic Knee Osteoarthritis. 2007;106(9).

10. Yanagisawa S, Ohsawa T, Saito K, Kobayashi T, Yamamoto A, Takagishi K. Morphological evaluation and diagnosis of medial type osteoarthritis of the knee using ultrasound. J Orthop Sci. 2014;19(2):270274. doi:10.1007/s00776-013-0524-9

11. Epis O, Paoletti F, D'Errico T, et al. Ultrasonography in the diagnosis and management of patients with inflammatory arthritides. Eur J Intern Med. 2014;25(2):103-111. doi:10.1016/j.ejim.2013.08.700

12. Podlipská J, Koski JM, Kaukinen P, et al. Structure-symptom relationship with wide-area ultrasound scanning of knee osteoarthritis. Sci Rep. 2017;7:44470. doi:10.1038/srep44470

13. Cubukcu D, Sarsan A and Alkan H. (2012): Relationships between pain, function and radiographic findings in osteoarthritis of the knee: a cross-sectional study. Arthritis; 2012:984060.

13. Zhang F, Kumm J, Svensson F,
Turkiewicz A, Frobell R, Englund M. Risk factors for meniscal body extrusion on MRI in subjects free of radiographic knee osteoarthritis: Longitudinal data from the Osteoarthritis Initiative. Osteoarthr Cartil. 2016;24(5):801-806. doi:10.1016/j.joca.2015.12.003

14. Kijima H, Miyakoshi N, Kasukawa Y, et al. Cut-off value of medial meniscal extrusion for knee pain. Adv Orthop ;2017. doi:10.1155/2017/6793026

15. Finan PH, Buenaver LF, Bounds SC, et al., (2013): Discordance between pain and radiographic severity in knee osteoarthritis: findings from quantitative sensory testing of central sensitization. Arthritis Rheum.; 65(2):363-372.

15. Kijima H, Yamada S, Nozaka K, Saito H, Shimada Y. Relationship between Pain and Medial Meniscal Extrusion in Knee Osteoarthritis. Adv Orthop ;2015. doi:10.1155/2015/210972

16. Kawaguchi, K., Enokida, M., Otsuki, R., \& Teshima, R. (2011). Ultrasonographic evaluation of medial radial displacement of the medial meniscus in knee osteoarthritis. Arthritis \& Rheumatism, 64(1), 173-180. doi: 10.1002/art.33319

To Cite This Article: Bishr DE, El-Fawal MA, El-Gerby KM , and El-Maghraby AM, Dynamic Ultrasound In Morphological Assessment Of Medial Compartment Of Knee Joint Osteoarthritis. ZUMJ 2019; 25 (5): 619-629. Doi: 10.21608/zumj.2019.7289.10260 